\title{
The Application Discussion of Big Data Technology in Intelligent Transportation
}

\author{
Yan Hou \\ Qilu Normal University, Jinan 250014 China
}

Keywords: Intelligent transportation; big data technology; Application.

\begin{abstract}
At present, the related data information with GPS positioning, road condition, traffic operation and video surveillance is gradually increasing in the construction process of intelligent transportation around all the cities of our country, and the produced data amount every day nearly has reached $\mathrm{PB}$ grade and presented the exponential growing trend. If so much data can be deeply mined and analyzed, it is bound to have unexpected effect on the traffic emergency management, society security. Thus, it is necessary to make promotion and application with big data technology. This paper made analysis and exploration with the application of big data technology in intelligent transportation.
\end{abstract}

\section{Introduction}

With the promotion of city development level in our country, the condition of traffic jam has been more and more serious. So faced with varieties of traffic problems, intelligent transportation comes into being. And intelligent transportation system integrates electric sensor technology and information technology, which can help to enhance the quality of transportation management to some degree. But it is the premise condition to achieve intelligent transportation construction that accurately and timely acquiring varieties of traffic data and construct the handling model of traffic data through big data technology.

\section{The Introduction of Big Data Technology}

\subsection{Concept of Big Data Technology}

Big data technology means one kind of technology that can quickly and accurately finds the valuable information from large amount of data, and it is the key point to solve all kinds of problems under the present big data circumstance. The one hand of challenge that big data technology is faced with shows that how to find worthy information from large amount of data, and the other hand also shows that how to make further increasing of the exploration and application of big data technology.

\subsection{The Composition of Big Data Technology}

Big data technology mainly includes the following several components: first, data collection, namely, making the cleaning and collective arrangement after abstracting the data from data source to the temporary intermediate level, and then loading them to the data mart or database. Second, data storage, including SQL, relation database, etc. Third, statistical analysis, including main component analysis and hypothesis testing. Fourth, data handling, in order to realize the handling with natural language, the most important thing is to make computer "understand" natural language. Fifth, basic structure, namely, cloud storage, etc. Sixth, model prediction, including predicting model and modeling and simulation. Seventh, data mining, namely, making the deep research with the classification, association rules and affinity grouping of data. Eighth, result presenting, including cloud computing and drawing relation diagram.

\section{The Characteristics of Big Data Technology}

The appearance of big data technology has brought great convenience for the construction of modern city intelligent transportation, which is mainly decided by the characteristics of big data technology. Specifically, big data technology mainly includes the following several aspects characteristics: first, high efficiency, big data technology has very high efficiency on data mining and 
can quickly find the existing internal relation and rules among large amount of traffic data, so that providing safeguard for the increasing of road passage ability and traffic operation management. Second, timeliness, big data technology can make timely and effective deep analysis and handling with large amount of data and quickly make response to help traffic management members to timely find the existing abnormal condition in the traffic operation and make correct traffic decisions. Third, predictability, big data technology has certain prediction function and can help users to master the traffic jam condition early to avoid the heavy-traffic road and make real-time dynamic monitoring with traffic condition. Fourth, distribution, big data technology can make distributed arrangement with data and make association analysis with complex model, provide service meeting multi-user visiting and offer help of handling traffic emergency case and multi-aspect coordination for people.

\section{The Application of Big Data Technology in Intelligent Traffic}

\subsection{The Application of Big Data Technology in Traffic Guidance}

There are many reasons for happening the problem of city road traffic, including big traffic flow, beyond the limit of road carrying capacity; imperfect traffic road basic facility construction; difficult parking and mess passage result in the sharp declining of road carrying capacity; the inconsistency of road traffic plan scheme and vehicle continuous increasing. Recent years, along with the increasing of city construction level, the phenomenon of occupying-road construction is more and more popular, which further intensifies the contradiction of road carrying capacity and traffic demand. Besides, the bad driving behaviors of drivers also aggravate the road congestion to some degree, and traffic guidance is one important method to solve traffic jam problem.

\subsubsection{The Shortcoming of Traffic Guidance and Traditional Traffic Guidance Technology}

Traffic guidance is a real-time dynamic process, needing to make use of varieties of technology methods to collect real-time traffic operation data, and data collection is the premise and basis to realize the traffic guidance. After collecting the needed traffic data, the current traffic condition should be made assessment and tried to make prediction with traffic in the shortest time through constructing traffic prediction model. Later, use TV, broadcast and radio to publish the guidance information and then get the guidance feedback result through the monitoring of traffic flow data to make promotion and optimization with guidance scheme. Viewing from the current situation, traditional traffic guidance technology cannot adapt the demand of times development, and its disadvantages mainly express from the following several aspects: first, limited by the region, most cities adopt the regional and sub-sector method to manage traffic, and due to lacking of communication and sharing of information among different sectors, it results in the collection of traffic data limited by the region. Second, limited by the data collection and storage. Most of traditional traffic data collection methods are random sampling, and most of collected traffic data are simulated data rather than number information, which is very bad for data storage. Third, the traffic flow monitoring system of most cities are only covering the main road of city, and the collected traffic data information is incomplete, which leads to the traffic flow judgement lacking of accuracy.

\subsubsection{The Advantages of Big Data Technology in Traffic Guidance}

In the aspects of traffic data collection and traffic flow monitoring, big data technology mainly has the following advantages: first, big data technology can make the whole monitoring with the entire traffic network system, rather than only collecting some sample data. Second, in the aspect of data collection, big data technology has more microcosm, which regards human as its objective to make the mobile data collection, and collected data includes phone GPS data and IC card data. Third, on data handling, big data technology can effectively avoid the disadvantages of simplification and inconvenience of storage of data pattern, making the original static data set convert to the data set that combines static with dynamic, and at the same time, it makes the improvement and innovation in the collection and conversion of simulated data. Fourth, it can realize the real-time observation with traffic condition and collect the traffic flow information through road monitoring technology, and it is convenient to make judgement with traffic operation condition. Meanwhile, the providing of dynamic data can also reflect the current traffic jam condition and traffic operation condition, making the prediction with the traffic flow during one period. Fifth, big data can collect the non-structural 
data related with traffic, including the social network diary, word and WEB click stream closely related with the daily traveling of people. For example, it can make the judgement with the traffic condition in its location through the WeChat and qq dynamics published to pedestrians.

\subsection{The Application of Big Data Technology in Safeguarding the Traffic Transportation Safety}

Along with the rapid development of domestic national economy, the traffic demand in all big cities is also sharply increasing and the traffic accident often happens. Road traffic safety has been the focus widely noticed by the entire society, and reducing the occurrence probability of traffic accidents and guaranteeing the security of road traffic have been the urgent demand of people in the current stage. In the road traffic system, due to the influence of factors, such as vehicle safety performance, environment, weather and driver quality, the traffic accident is easy to happen, and the predictability of big data technology and timeliness performance can make active forewarning with accidents in order that the related members early understand the occurrence probability of accidents. For example, using GPS positioning technology to collect the related information of vehicle traveling track can make judgement whether the motor is in the normal operation condition, and once finding the abnormal condition, the traffic department can be timely informed to control the car. For another example, using road environment and facility supervision system can make timely collection with the dynamic information of road environment and facility, and publish to the traffic radio platform or send to the car in the related driving region with the pattern of mobile phone message after the cloud computing handling. In addition, combining the big data technology with emergency aid system can more accurately judge the correct location of accident occurrence and timely inform the fire rescue and nursing team, also sending the news of happening accidents to the cars nearby to make it easy to prepare giving way for rescue vehicles.

\subsection{The Application of Big Data Technology in Optimizing the Public Transportation Service}

Public transportation means that the transportation methods of rail transit, bus and cableway that are operated on the pointed routes in the city region, and all of these transportation tools depart at fixed time point. Once the resource allocation is irrational, it will result in many problems, for example, the waiting time of passengers is too much and the crowed train. However, the application of big data technology can further increase the reasonability of resource allocation and more rationally allocate the public resource and promote the resource usage through the real-time monitoring with the passenger flow volume of station. In the meanwhile, passengers can use mobile APP to make the real-time query with the operation condition of bus, and it can offer the related information of passengers flow volume condition in the car to passengers in order to make passengers timely adjust their train plan, avoiding the occurrence of blindly taking bus. Public transportation is a significant method to improve the city traffic jam, and it can strengthen the public transportation service level, making citizens truly feel the convenience brought by the public transportation, and it is one premise condition of citizens choosing public transportation to travel.

\subsection{The Application of Big Data Technology in the Active Intelligent Transportation Service 4.4.1 Active High-Speed Rail Train System}

High-speed rail is one of the important traffic tools in the modern city, and in order to catch the high-speed rail, passengers often go to the high-speed rail station a few hours in advance, and the time to catch the rail even far exceeds the time to take high-speed rail. Applying the big data technology to the transportation, tax drivers can cooperate with the high-speed transportation department and acquire the phone number and train time of passengers in advance, and make prediction with the required time of passengers from the start point to the high-speed rail station, send the best route and train method to passengers with the big data technology handling technology. If passengers choose to take taxi, then they can use the GPS positioning technology of mobile phone to acquire the taxi information near the pick-up point at proper time, and taxi drivers can arrive at the station by the optimal path in the shortest time through the real-time transportation information service, which can save much catching train time for passengers.

\subsubsection{Active Parking System}

Along with the continuous increasing of vehicles amount, the problem of difficult parking has been paid more and more attention to by people, and solving difficult parking problem is one 
important method to manage the city traffic jam, and applying the big data technology to the intelligent transportation system can actively send the related transportation service information to users. For instance, by means of electronic license plate GPS positioning technology, the parking time and specific location of cars can be timely acquired, and once finding the condition of parking breaking the rules, it can send the car owner the related illegal information and make the owner drive the car in the shortest time, which can solve the problem of traffic jam due to the mess parking of vehicles. Using parking guidance system also can acquire the location of vehicle and the parking lot information near its nearby region and predict the time to arrive at the nearest parking lot, then it will send the related parking lot information to the car owner through mobile APP or message in order to make it easy for car owners to choose the parking site and order the parking spot in advance.

\section{Conclusion}

In conclusion, the construction of intelligent transportation system is the inevitable trend of modern transportation industry development, and it also is the revolutionary transition of traffic industry. And the application of big data technology provides the possibility for the achievement of intelligent transportation, and it further enhance the automation, integration and information level of intelligent transportation. Through the analysis with the concept, constitution and characteristic of big data technology, this paper deeply discussed the application of big data technology in all the aspects of intelligent transportation, for example, traffic guidance, traffic transportation security, public transportation service and active intelligent transportation service, which fully proved that the application possibility and necessity of big data technology in such industries. In the future, the application of big data technology in the intelligent transportation will be bound to involved to the big data handling technology, for example, cloud computing, but using this technology needs to construct a mass data platform, which will effectively solve the problem of mass data explosion. Therefore, the construction to mass data platform must be further strengthened in the future work, and do better with the work of systematic structure, data storage scheme and data transition plan. Only by this way, can a more convenient and effective intelligent transportation system be created.

\section{References}

[1]. Jinbin Chen. The application of big data technology in intelligent transporation [J]. Goods and quality, 2016,(42):404.

[2]. Xiang Gong, Wei Qi, Zuoxin Wang. The application of big data technology in intelligent transporation[C].//The fourth Chinese intelligent transportation congress and collection. 2013:18-23.

[3]. Jing Yang, Xiao Zhang, Liqun Wang. The application of big data technology in intelligent transporation [J]. Techonoly Wind, 2015,(19):110.

[4]. Hongen Dang, Erping Zhao. The application of big data technology in intelligent transporation [J]. Wireless internet technology, 2017,(2):147-148.

[5]. Jianguo Li. The application of big data technology in intelligent transporation [J]. Technology and enterprise, 2015,(7):73-73.

[6]. Lingling Yang, Fasheng Liu, Xia Dong. The application of big data technology in intelligent transporation[J]. Chinese market, 2016,(23):154-156.

[7]. Li Xu, Haiyuan Xia. The application of big data technology in intelligent transporation [J]. China security and service, 2016,(5):67-69. 